Brit. J. vener. (1956), 32, 188.

\title{
OCCURRENCE AND ECOLOGY OF MYCOPLASMA SPECIES (PLEUROPNEUMONIA-LIKE ORGANISMS) IN THE MALE URETHRA*
}

BY

\author{
E. A. FREUNDT
}

From the Statens Seruminstitut, Copenhagen (Chief: J. Ørskov, M.D.), and the Dermato-Venerological Department of Københavns Militaer-Hospital (Chief: S. Emanuel, M.D.)

Since the discovery by Dienes, Berg, and others (Dienes and Edsall, 1937; Dienes, 1940; Dienes and Smith, 1942, 1946) that Mycoplasma species $\dagger$ (pleuropneumonia-like organisms) are frequent inhabitants of the male and female genital tracts, the possible role of these organisms in non-gonococcal urethritis (NGU) has been the subject of much research. The incidence of Mycoplasma species in NGU reported on by previous authors ranges from 7 to 53 per cent.: Beveridge (1943), 17 per cent.; Beveridge, Campbell, and Lind (1946), 20 per cent.; Salaman (1946), 7 per cent,; Harkness (1950), 17 per cent.; Melén and Linnros (1952), 18 per cent.; Nicol and Edward (1953), 26 per cent.; Wagner, Morse, and Kuhns (1953), 12 per cent.; Jensen (1954), 22 per cent.; Durel, Roiron-Ratner, Siboulet, and Sorel (1954), 14 per cent. and Shepard (1954), 53 per cent. In patients with acute gonorrhoea, Harkness (1950) found the same percentage as with NGU (17 per cent.), while Salaman (1946) found a higher, and Nicol and Edward (1953) and Melén and Linnros (1952) a lower incidence $(34,11$, and 3 per cent. respectively). The results obtained in apparently healthy men are even more conflicting. Beveridge and others (1946) and Harkness and HendersonBegg (1948) failed to isolate mycoplasmata from 67 and fifty normal controls respectively. However, values ranging from 10 to 36 per cent. were reported by Salaman (1946), Dienes and others (1948), Bushby (in Harkness, 1950), Melén and Linnros (1952), Nicol and Edward (1953), and by Durel and others (1954). Finally, Shepard (1954), in three population groups with different sexual activity, found 56, 33, and 2 per cent. Although in recent years there has been increasing evidence that the urethral mycoplasmata are in most cases mere saprophytes or commensals, further investigation in support of this view may still be of interest, both from a clinical and an ecological point of view.

\footnotetext{
* Received for publication April 11, 1956

+ In the following, the terms Mycoplasma species or mycoplasmata will be used for "pleuropneumonia-like organisms" (PPLO) according to the terminology, introduced by Freundt (1955), Edward (1955), and Edward and Freundt (1956).
}

MATERIAL

The investigation was mainly carried out between 1950 and 1952 on military personnel, comprising 195 men attending the out-patient clinic or admitted to the skin and venereal diseases department of the Military Hospital, Copenhagen.f In addition, 45 patients (with gonorrhoea) attending various out-patient clinics in Copenhagen were examined in 1955. The total material of 240 men comprised five clinical groups (Table).

\section{TABLE}

ISOLATION OF MYCOPLASMA SPECIES (PLEUROPNEUMONIA-LIKE ORGANISMS) FROM THE MALE URETHRA

\begin{tabular}{|c|c|c|c|}
\hline \multirow{2}{*}{ Diagnosis } & \multirow{2}{*}{$\begin{array}{l}\text { No. of } \\
\text { Patients }\end{array}$} & \multicolumn{2}{|c|}{ Mycoplasma species } \\
\hline & & No. & Per cent. \\
\hline $\begin{array}{l}\text { Non-gonococcal urethritis } \\
\text { (NGU) }\end{array}$ & 109 & 33 & \\
\hline $\begin{array}{llr}\text { Subclinical NGU } & \text {. } \\
\text { Acute gonorrhoea } & \ldots \\
\text { Epididymitis without urethritis } \\
\text { No genital abnormality }\end{array}$ & $\begin{array}{r}30 \\
50 \\
23 \\
28\end{array}$ & $\begin{array}{l}33 \\
15 \\
11 \\
15\end{array}$ & $\begin{array}{l}50 \cdot 0 \\
34 \cdot 0 \\
47 \cdot 8 \\
53 \cdot 6\end{array}$ \\
\hline Totals .. & 240 & 91 & $37 \cdot 9$ \\
\hline
\end{tabular}

(1) Non-gonococcal Urethritis (NGU) was diagnosed in 109 patients. Fifteen of these reported previous attacks of NGU with a symptom-free interval of at least 6 months, and 21 gave a history of gonorrhoea one or more years earlier. 21 cases had acute epididymitis, but other complications such as prostatitis, conjunctivitis, and joint symptoms were rarely found. The age of the patients ranged from 17 to 44 years (average 22).

(2) Subclinical NGU.-A diagnosis was made on the basis of cytological findings in thirty patients who showed no evidence of urethral infection on inquiry and clinical examination. This group includes 22 out of fifty men, originally selected as normal controls, with skin complaints, and eight patients admitted for acute epididymitis. Their ages ranged from 20 to 27 years (average 21).

$¥$ My thanks are due to Dr. S. Pindborg, who performed the clinica examination of the patients and took the urethral specimens. 
(3) Acute Gonorrhoea was diagnosed in fifty patients whose ages ranged from 17 to 47 years (average 27).

(4) Acute Epididymitis without Urethritis was diagnosed in 23 patients who gave no history of recent gonococcal infections. The gonococcal complement-fixation test in these and other cases of epididymitis was negative on repeated examination. A tuberculous aetiology appeared to be unlikely from the clinical findings and rather benign course of the disease. Their ages ranged from 18 to 44 years (average 21).

(5) Controls. -28 of the above-mentioned fifty men with skin complaints were finally selected as controls. The following diagnoses were represented: verrucae, hyperkeratosis plantae, hyperhidrosis, naevi, defluvium capillarum, pityriasis capitis, eczema of the hands or feet, epidermophytia pedis, preventive scabies therapy, zoster, furunculosis, acne faciei, pityriasis rosea (one case), and psoriasis (one case). Their ages ranged from 20 to 40 years (average 22 ).

\section{Methods}

Smears made from repeated urethral scrapings of patients with urethritis were examined for gonococci in the clinic. In addition, on the first examination in several cases (including all the controls), smears were sent to the bacteriology laboratory. They were then stained with Giemsa overnight and examined for bacteria, leucocytes and epithelial inclusion bodies. Control examinations showed that the number of bacteria that could be recognized in smears stained with Giemsa was similar to that found in Gram-stained smears.

Specimens for cultivation were taken from the urethral orifice by means of sterile cotton-wool swabs, which were immersed in a transport medium consisting of soft "chocolate" agar, and they were sent to the laboratory. Inoculation on to the culture media was generally made about 3 to $6 \mathrm{hrs}$ after the specimens had been taken.

Cultivation for gonococci was made repeatedly in every case of urethritis or epididymitis (performed in the Statens Seruminstitut).

Cultivation for other bacteria was made on blood agar by aerobic and anaerobic incubation. McLeod's tellurite medium was used for the isolation of corynebacteria.

Cultivation for Trichomonas vaginalis was made in a composite medium previously found to support an excellent growth of $T$. vaginalis from a high percentage of women (Freundt, 1953).

Cultivation for Mycoplasma species was made from one to seven times in each case of genital disease and once in each of the controls.

Two kinds of solid media and a semi-solid medium were used:

(a) A solid medium prepared from human placenta infusion containing 1.5 per cent. agar, 2 per cent. Bactopeptone, and 30 per cent. ascitic fluid, with further addition of penicillin ( 2 units $/ \mathrm{ml}$.) and thallium acetate $(1: 2000)$. One plate was incubated aerobically, another anaerobically, both of them in jars containing about 10 per cent. $\mathrm{CO}_{2}$ (Freundt, 1953).

(b) Solid ascitic medium without bacteriostatics. Aerobic and anaerobic incubation in a $\mathrm{CO}_{2}$ atmosphere.

(c) A semi-solid infusion broth medium containing about $0 \cdot 2$ per cent. agar, 20 per cent. ascitic fluid, 1 per cent. yeast extract, penicillin ( 2 units $/ \mathrm{ml}$.), and thallium acetate $(1: 2000)$.

The swabs were inoculated both on the plates (four plates for each swab) and on the semi-fluid medium. After incubation of the latter for 2 days, a subculture was made on solid medium without bacteriostatics.

The plates were examined microscopically for mycoplasma-colonies after 2 and 4 days' incubation, using a low-power lens and transmitted light.

Attempts were made to improve the cultivation results by special procedures. Duplicate urethral swabs were frequently taken; the first one was streaked immediately on to solid ascitic medium, and inoculation from the second one was made 3 to 6 hours later as usual.

Cultivation was also made in several cases from prostatic secretion obtained by rectal massage of the prostate and vesiculae seminales. The fluid was withdrawn from the urethral orifice by means of a small capillary tube. Although by this method no information could be obtained as to a possible primary occurrence of mycoplasmata in the secretion, it was thought that the outflow of the viscous fluid might serve to irrigate the urethra and thereby afford a better material for obtaining growth of the organisms than the ordinary swabs. (For comparison, urethral swabs were taken from the same patient just before the prostatic massage.)

As regards the 45 patients with gonorrhoea examined in 1955 , the investigation was restricted to cultivation for mycoplasmata. For a fortnight all swabs from men in Copenhagen sent to the Gonococcus Department were immersed in the above-mentioned semi-solid medium immediately after inoculation on the gonococcal medium. Subcultures from the semi-solid on to the solid media were made after 2 days' incubation in those cases that had meanwhile yielded a growth of gonococci.

\section{Results}

\section{Bacteriological Examination}

NGU is frequently classified as bacterial or abacterial on the basis of urethral smears and/or cultures (Harkness, 1950). However, recognized pathogens are seldom found in the former group, and the correctness of maintaining the two types as clinical entities was doubted by Ambrose and Taylor (1953) and Willcox (1954a). In the present work, Giemsa-stained urethral smears were examined from 163 patients (88 with NGU, thirty with subclinical NGU, and 45 without urethritis-including the 28 controls and seventeen cases of epididymitis). The smears could easily be classified into two groups: those revealing no or few bacteria, and those in 
which many or even myriads of cocci and rods were found throughout the preparation. Rather surprisingly, the former type of smear was more frequently obtained from patients with clinical NGU than from the other groups:

\begin{tabular}{|c|c|c|c|c|}
\hline Diagnosis & & $\begin{array}{c}\text { "Abacterial" } \\
\text { Smears }\end{array}$ & $\begin{array}{l}\text { Total } \\
\text { Smears }\end{array}$ & $\underset{\text { (per cent.) }}{\text { Smears }}$ \\
\hline $\begin{array}{l}\text { NGU } \\
\text { Subclinical NGU } \\
\text { No urethritis }\end{array}$ & $\begin{array}{l}\cdots \\
\cdots \\
\cdots\end{array}$ & $\begin{array}{r}66 \\
6 \\
15\end{array}$ & $\begin{array}{l}88 \\
30 \\
45\end{array}$ & $\begin{array}{l}75 \\
20 \\
33\end{array}$ \\
\hline
\end{tabular}

In NGU, the frequency of abacterial smears appeared to increase with increasing duration of the urethritis (Figure, p. 192).

Cultivation for bacteria other than gonococci was made one or several times in 79 patients (55 with NGU, eight with subclinical NGU, three with gonorrhoea, and thirteen without urethritis). The number of bacteria found on cultivation corresponded fairly well with the smears, although growth was sometimes heavier than might have been expected from the smears. Whether growth was sparse or heavy, staphylococci ( $S$. albus) and corynebacteria were isolated in almost every case. At least three colony-types of the latter were observed on tellurite medium; they could not be identified with named species by fermentation reactions or toxigenicity tests. Other bacteria, each found only on a few occasions, were $S$. aureus, $\alpha, \beta$, and $\gamma$ streptococci, enterococci, Gram-negative rods of the Escherichia and Klebsiella groups, $H$. influenzae, Neisseriae other than $N$. gonorrhoeae, Bacteroides, anaerobic staphylococci and streptococci. The range of bacteria found in patients with genital disease was similar to that found in the controls, and no doubt the vast majority were saprophytes or accidental commensals. Trichomonas vaginalis was not found in any of 26 patients with NGU examined for this organism. Indisputable epithelial inclusions were demonstrated in only three cases of NGU (and one of gonorrhoea), an incidence corresponding to that reported by Siboulet (1955) in a survey of 2,756 cases of NGU.

\section{Mycoplasma SPECIES}

Cultures from 365 sets of swabs were examined, and growth of Mycoplasma species was obtained on 119 occasions from 91 individuals. There appeared to be no risk that L-phase variants of bacteria induced by the low concentration of penicillin contained in one of the media might have been confused with mycoplasmata, as mycoplasma-colonies of similar appearance were generally present on plates both with and without bacteriostatics. Discrepancies were found only in the case of very sparse growth, or when the plates without bacteriostatics were over. grown by bacteria. Growth of mycoplasmata was exclusively found on the anaerobic plates in thirteen, and on the aerobic plates in six cases. In most of these cases the growth was very sparse, and the discrepancy may largely be due to mere chance. However, in subcultures also, the growth of some strains (later on identified as Mycoplasma fermentans) was definitely enhanced by anaerobic incubation. Cultivation in semi-solid medium with subsequent subculturing on plates did not increase the number of positives to any considerable degree: only in four cases was growth exclusively obtained from the semi-solid culture. Duplicate urethral swabs were taken on 67 occasions. The results gained by the immediate inoculation of the swabs on to solid medium were no better than those obtained from the swabs after storage for some hours. Cultivation from prostatic secretions of 23 patients with epididymitis yielded growth of mycoplasmata in two cases that were negative from the swabs, while the opposite result was found in three cases. However, growth was frequently more abundant from the prostatic secretions than from the swabs (Table).

Non-Gonococcal Urethritis.-Mycoplasma species were isolated in 33 (30.3 per cent.) of 109 cases of NGU (Table). The same incidence was found in cases with or without epididymitis. The growth was sparse in 23 , moderate in four, and heavy in only six cases.

From the character and macroscopic appearance of the discharge, the patients could roughly be divided into two groups: one characterized by a rather sparse, serous or mucoid to slightly mucopurulent discharge, possibly occurring only as a "morning drop" (63 cases), and another group with a more abundant, more or less purulent discharge (46 cases). However, there was no difference in the occurrence of mycoplasmata between the two groups (28.9 and 30.4 per cent., respectively). In agreement with this observation, no correlation could be found between the number of leucocytes in smears and the occurrence of mycoplasmata: the incidence was the same whether very few or innumerable leucocytes were found in the discharge.

Presumably reliable information about the previous duration of symptoms at the time when the patients were first examined for mycoplasmata was obtained in 96 cases. In 64 cases in whom symptoms had been present from 1 to 21 days, mycoplasmata were found in 23 (35.9 per cent.). 32 patients gave a history of symptoms lasting from 1 month to 5 years, and only four of these $(12.5$ per cent.) were positive. 
The different incidence in these two groups is statistically significant $(0.02<P<0.03)$.

Unfortunately, the different kinds of therapy that were attempted in the present series of NGU with or without occurrence of mycoplasmata were not planned and carried out systematically with proper control groups. Consequently, no conclusion as to the aetiological significance of the organisms could be drawn from the over-all results of treatment. However, certain observations in this connexion appeared to be of some interest. Mycoplasmata are highly susceptible to aureomycin. Most mycoplasmapositive cases treated with this antibiotic responded promptly with clinical recovery, and at the same time the mycoplasmata disappeared from the urethra. But of course, this in itself does not allow any conclusions to be drawn as to the aetiological implication of the organisms. However, in one patient growth was still obtained after treatment, while on the other hand discharge and dysuria had temporarily disappeared. Eight days later a relapse occurred: the patient was then treated with chloramphenicol, after which the mycoplasmata could not be recovered; but this therapy had no effect on symptoms. Thus, in this patient there was clearly no relation at all between the occurrence of mycoplasmata and the symptoms. Another patient gave three negative swabs during urethritis characterized by abundant purulent discharge that disappeared spontaneously after 5 weeks. On examination 20 days after discharge from the hospital, he was still found to be quite healthy, but a urethral swab yielded growth of mycoplasmata. In seven patients the mycoplasmata apparently disappeared spontaneously during the course of the disease. In two cases the disappearance of the organisms was accompanied by complete clinical recovery, while in the other five no definite improvement was recorded until later on.

Subclinical Non-Gonococcal Urethritis and Related Conditions of the Genito-Urinary Tract.-Among the fifty men primarily selected as normal controls a surprisingly high number of polymorphonuclear leucocytes was found in urethral smears from no less than 22 persons; numbers of four to ten cells occurring in several high-power fields (oil-immersion lens) were found in nine cases, and even more numerous leucocytes (up to fifty or seventy, or innumerable cells per field) were demonstrated in thirteen cases.

The strictly normal urethral mucosa, as well as the secretions of the para-urethral and bulbo-urethral glands and the prostatic secretion, are generally said to contain very few polymorphonuclear leucocytes
(Stieve, 1930). Ambrose and Taylor (1953), somewhat arbitrarily, estimated less than ten leucocytes per field (high-power, dry objective) for the normal prostatic secretion. The finding of numerous leucocytes probably indicates the presence of a subclinical NGU or some other mildly inflammatory condition of the genito-urinary tract (Harkness, 1955). Although the significance of counts of four to ten cells is perhaps more dubious, persons yielding such smears were also excluded from the controls and classified as cases of subclinical NGU.

It is known that certain skin diseases may be complicated by lesions on the urethral mucous membrane (Harkness, 1950). However, none of the skin diseases mentioned by Harkness in this connexion were represented in the present material, and certainly in the vast majority of the above cases any connexion between urethritis and skin disease would appear to be out of the question.

In addition to the cases of skin disease, eight patients admitted with acute epididymitis presented signs of presumed subclinical urethritis in the form of leucocytes in urethral scrapings. Mycoplasma species were isolated in fifteen (50 per cent.) of the thirty cases thus classified as subclinical NGU and related conditions. The growth was sparse in four, and moderate to heavy in eleven cases.

Gonococcal Urethritis.-In this group of fifty patients, Mycoplasma species were demonstrated in seventeen (34 per cent.).

Epididymitis without Urethritis. - 23 patients suffering from acute non-gonococcal epididymitis had no previous or present symptoms of urethritis. In seventeen cases, only occasional leucocytes were found in urethral scrapings, while no smears were available from six. Although a few of the latter may have had subclinical urethritis, this would not appear to affect the evaluation of the results to any great extent.

Mycoplasma species were obtained from the urethral orifices of eleven $(47 \cdot 8$ per cent.) of the patients in this group (sparse growth in four, and moderate to heavy growth in seven).

A puncture of the swollen epididymis was made in one patient (with negative urethral cultures). Cultivation of the puncture fluid, and from the pus of an abscess which developed later, yielded $E$. coli only. In two other cases of epididymitis not included in the present material, one of which gave a positive swab culture, no growth at all was obtained from the puncture fluid. 
Normal Controls. -28 men with various trivial skin complaints showed no clinical evidence of recent genital infection, and no, or only occasional, leucocytes were demonstrated in urethral smears. These men could thus be considered strictly healthy as regards their genitals. Sparse growth of Mycoplasma species was obtained from four, and moderate to heavy growth from eleven ( 53.6 per cent.).

\section{Relations of Mycoplasma Species to Bacterial FLORA}

In the Figure, the mycoplasma-positive cases have been grouped according to the number of bacteria demonstrated in urethral scrapings stained with Giemsa from a total of 163 men representing patients with clinical and subclinical NGU and individuals with an apparently healthy urethral mucosa. It will be seen that, regardless of the clinical diagnosis, a definite correlation is found between the occurrence of Mycoplasma species, and the number of ordinary bacteria, the incidence of mycoplasmata invariably being significantly higher in groups of individuals characterized by smears rich in bacteria. Out of a total of 87 males yielding relatively "abacterial" smears, Mycoplasma species were isolated from only fifteen (17.2 per cent.), while 49 mycoplasmapositive cases (64.5 per cent.) were found among

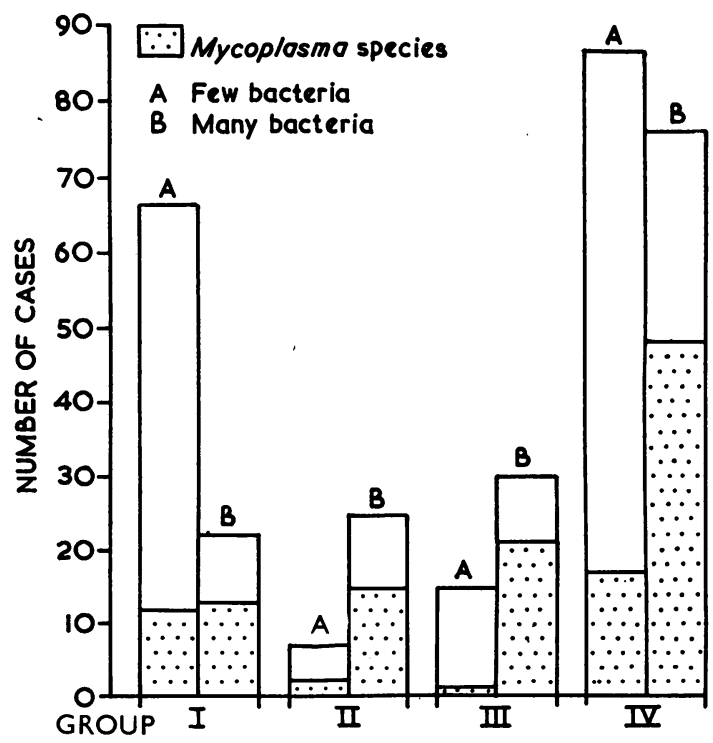

Figure.-Occurrence of ordinary bacteria in urethral smears from 163 patients with and without non-gonococcal urethritis, and relation of Mycoplasma species to number of bacteria.

I. -88 patients with non-gonococcal urethritis.
II. -30 patients with subclinical non-gonococcal urethritis

III.-28 normal controls and seventeen epididymitis-patients with

apparently healthly urethral mucosa.

IV.-Groups I to III combined.
76 patients apparently harbouring large numbers of bacteria at the external urinary meatus and the fossa navicularis.

\section{Classification and Nomenclature of Human Genital Mycoplasma SPECIES}

In a preceding paper (Freundt, 1954), human strains of the Mycoplasma group were classified into two main groups (I and II). The properties previously described for these groups are supplemented here with an examination of the growth characteristics on horse serum and rabbit serum agar that were used by Edward (1954) in the differentiation of species. It was then shown that the strains of Group II produced a pearly film and black spots on horse serum agar, and rabbit serum was found to support a good growth of both groups. Though serological identification has not yet been made, there seems to be little doubt that Group I is closely related to, or identical with, the strains designated by Nicol and Edward (1953) as Human Types 1 and 2, while Group II appears to be identical with Edward's Human Type 3. Recently, the name Mycoplasma hominis has been proposed for Types 1 and 2 , and $M$. fermentans for Type 3 (Freundt, 1955; Edward, 1955; Edward and Freundt, 1956). The name Micromyces hominis, originally proposed for Groups I and II (Freundt, 1954) has thus been abandoned.

Among the 91 strains isolated in the present work, only 57 were classified because the remaining strains were lost before appropriate methods for species identification had been worked out. The majority of the preserved strains conformed to the criteria set up for Mycoplasma hominis, and only eight strains were identified as $M$. fermentans, one of which had been isolated from a case of NGU, two from cases of subclinical NGU, three from cases of gonorrhoea, and two from normal persons.

\section{Discussion}

The results of the present investigation add to the increasing evidence obtained in recent years that Mycoplasma species are frequently part of the normal flora of the urethral orifice and the prepuce, and that their role in NGU is quite negligible.

Although the percentage of positive isolations $(30 \cdot 3)$ from patients with NGU was just as high or higher than that reported by most other authors, it was nevertheless lower than the incidence found in normal controls; both of the two species that were recognized occurred in patients with genital disease as well as in the controls. Species classification of a large number of strains isolated from men with and 
without NGU has previously only been made by Nicol and Edward (1953), who found all except one of 91 strains (including strains from females) to belong to one species (M. hominis, Type 1). The observation of Ruiter and Wentholt (1952) that the occurrence of $M$. fermentans (" $G$ " strains) was confined to ulcerative lesions of the glans penis disagrees with the present findings.

No relationship could be demonstrated between the occurrence of mycoplasmata and the clinical type of urethritis and the character of the discharge. This is in agreement with the experience of most other authors (Melén and Linnros, 1952; Nicol and Edward, 1953; Durel and others, 1954; Shepard, 1954). Nicol and Edward and Durel and others were not able to confirm the observation made by Harkness and Henderson-Begg (1948) and Harkness (1950) that mycoplasmata were more frequently associated with cases classified as "abacterial" urethritis than with the "bacterial" group of NGU. The close correlation found in the present investigation between mycoplasmata and the presence of abundant bacteria in urethral smears is apparently even more in conflict with the findings of Harkness and Henderson-Begg.

Apart from a remarkably lower incidence of isolations in cases of long duration, the relation of mycoplasmata to the course of the disease appeared to be rather fortuitous. Disappearance of the organisms, spontaneously or as a result of treatment, was not invariably accompanied by clinical improvement; they might on the other hand persist in the urethra in spite of successful therapy, or even appear for the first time after complete cure. Similar observations were made by Nicol and Edward (1953) and by Willcox (1954b).

The lower frequency of mycoplasmata found in patients with NGU than in the control group, together with the observations of a further decrease in the incidence of the organisms during the course of the disease, deserves special consideration.

On comparing the controls with the NGU patients it should first be noted that both groups appear to be equal as to age and social and occupational levels. The highly conflicting cultivation results obtained from normal men by different workers have previously been ascribed by Melén and Linnros (1952) and by Shepard (1954) to different socio-economic and hygienic levels of the population-groups examined.

The difference between the incidence of mycoplasmata in the controls (fifteen out of 28) and in the patients with NGU (33 out of 109) is statistically significant $(0.03<P<0.04)$. It should also be pointed out that in 23 epididymitis patients without urethritis the same percentage of mycoplasmata $(47 \cdot 8)$ was found as in the controls. The significance of the high incidence found in normal persons is moreover supported by the observation that individuals presenting only a mild, non-exudative, inflammatory reaction ("subclinical NGU") yielded an equally high percentage of positive isolations. The findings in patients with acute gonorrhoea (mycoplasmata obtained from 34 per cent.) are in accordance with those in acute NGU. Finally, it is worthy of note that in the present material sparse growth was more frequently obtained in the NGU group (in 23 out of 33 positive isolations) than in men without urethritis (in eight out of 26) or with only a subclinical urethritis (in four out of fifteen). This difference becomes even more obvious when it is considered that the material available for cultivation is much more abundant in the case of a urethritic exudate than when dealing with a scraping from the relatively dry, normal urethral mucosa.

The significance of the observation of a definitely lower incidence of mycoplasmata in chronic NGU than in acute cases is supported by the findings of Harkness (1950, p. 122) who reported "a much higher percentage of positive cultures" in the acute phase of NGU in Reiter's syndrome than later on.

It would thus appear that while in the normal urethra the conditions are fairly favourable for the prevalence of mycoplasmata, the organisms are probably depressed during inflammation, whether this be caused by gonococci or other agents, and the more so the longer the urethritis has lasted. The cause of a possible depression of the mycoplasmata remains obscure; it apparently cannot be ascribed to the migration of leucocytes, as no correlation was found between mycoplasmata and the number of leucocytes. A reduced chance of transmission of the organisms from female consorts as a result of a possibly restricted sexual activity during relatively long-lasting urethritis may also play a part, but this has not been investigated by questioning the patients about their sexual habits.

The whole problem should probably be considered in relation to the general bacteriological findings. Judging from the examination of stained smears, a similar quantitative change of the normal bacterial flora occurs during urethritis. The frequent coincidence between the occurrence of Mycoplasma species and numerous saprophytic bacteria lends further support to the assumption that mycoplasmata are merely part of the normal microbial flora of the urethral orifice and the fossa navicularis in sexually active men.

In females, a similar close relationship between mycoplasmata and a profuse vaginal flora was 
previously demonstrated (Freundt, 1954), but here the accompanying bacteria and protozoa were not normal inhabitants of the vagina. However, the situation in the normal vagina and on the male urethral mucosa cannot be directly compared, in so far as the growth conditions yielded by the normal, strongly acid vaginal secretion are decidedly unfavourable both to mycoplasmata and to nonacidophilic bacteria. The fact that a close correlation between the presence of mycoplasmata and gonococci has frequently been found in the female but not in the male (Nicol and Edward, 1953; Somerson, Rubin, Smith, and Morton, 1955), also suggests that the ecological relationships between the micro-organisms of the genitals are different in females and males.

The above considerations are open to the objection that an incidence of mycoplasmata in normal men exceeding that found in NGU-patients has not been demonstrated by other authors.

It is rather difficult, however, to compare groups examined by different investigators, because they may differ considerably in their composition. In addition to the factors previously mentioned (age, sexual activity), differences such as the criteria used for selecting normal controls, or the stage of disease at which cultivation has been made from patients with NGU, may greatly influence the results; e.g., in the latter case if the majority of the patients were examined in a very early phase of urethritis, almost the same percentage of mycoplasmata might be found as in normal men.

\section{SUMMARY}

Mycoplasma species (pleuropneumonia-like organisms) were isolated from the urethra of 30 per cent. of 109 men with non-gonococcal urethritis (NGU). The incidence was significantly lower in the chronic stage than in the acute phase of the disease. No relationship could be demonstrated between the occurrence of mycoplasmata and the symptoms and course of the urethritis.

In fifty men with acute gonorrhoea there was a similar frequency of mycoplasmata (34 per cent.) to that found in NGU.
A significantly higher incidence was demonstrated in thirty cases classified as subclinical NGU (50 per cent.), in 23 patients suffering from epididymitis without any signs of urethritis ( $47 \cdot 8$ per cent.), and in 28 men with no genital abnormality at all (53.6 per cent.).

A rather close relationship was found between the presence of mycoplasmata in cultures and numerous ordinary bacteria in urethral smears.

Most of the isolated strains of the Mycoplasma group were classified as Mycoplasma hominis, while eight strains were identified with $M$. fermentans. Both species occurred in all the clinical groups.

Mycoplasmata are apparently common saprophytes of the normal male urethra, and it is suggested that the lower incidence found in the present material in patients with clinical NGU and with gonorrhoea reflects a general suppression of the normal microbial flora as a result of inflammation.

\section{REFERENCES}

Ambrose, S. S., and Taylor, W. W. (1953). Amer. J. Syph., 37, 501 Beveridge, W. I. B. (1943). Med. J. Australia, 2, 479.

, Campbell, A. D., and Lind, P. E. (1946). Med. J. Aust., 1, 179. Dienes, L. (1940). Proc. Soc. exp. Biol. (N.Y.), 44, 468.

, Berg, R. L., and Weinberger, H. J. (1948). Ann. rheum. Dis., 7, 259 .

, and Edsall, G. (1937). Proc. Soc. exp. Biol. (N.Y.), 36, 740.

and Smith, W. E. (1942). Ibid., $50,99$.

$\longrightarrow$ (1946). J. clin. Invest., 25, 911 .

Dienes, L., Berg, R. L., and Weinberger, H. J. (1948). Ann. rheum. Dis., 7, 259.

Durel, P., Roiron-Ratner, V., Siboulet, A., and Sorel, C. (1954). British Journal of Venereal Diseases, 30, 69.

Edward, D. G. ff. (1954). J. gen. Microbiol., 10, 27. (1955). Int. Bull. bact. Nomencl., 5, 85 .

- , and Freundt, E. A. (1956). J. gen. Microbiol., 14, 197.

Freundt, E. A. (1953). Acta path. microbiol. Scand., 32, 468.

(1954). Ibid., 34, 127.

(1955). Int. Bull. bact. Nomencl., 5, 67.

Harkness, A. H. (1950). "Non-Gonococcal Urethritis". Livingstone, Edinburgh.

(1955). Personal communication.

and Henderson-Begg, A. (1948). British Journal of Venerea

Diseases, 24, 50.
Jensen, T. (1954). Amer. J. Syph., 38, 125.

Melén, B., and Linnros, B. (1952). Acta derm.-venereol. (Stockh.), 32, 77.

Nicol, C.'S., and Edward, D. G. ff. (1953). British Journal of Venereal Diseases, 29, 141

Ruiter, M., and Wentholt, H. M. M. (1952). J. invest. Derm., 18, 313.

Salaman, M. H. (1946). J. Path. Bact., 58,31.

Shepard, M. C. (1954). A. Amer. J. Syph., 38, 113.

Siboulet, A. (1955). British Journal of Veneral Diseases, 31, 235.

Somerson, N. L., Rubin, A., Smith, P. F., and Morton, H. E. (1955). Amer. J. Obstet. Gynec., 69, 848 .

Stieve, H. (1930). In "Handbuch der mikroskopischen Anatomie des Menschen", Bd. VII/2, pp. 246, 267, 268. Ed. W. von Mollendorff. Springer, Berlin.

Wagner, B. M., Morse, W. H., and Kuhns, D. M. (1953). Amer. J. publ. Hith, 43, 853 .

Willcox, R. R. (1954a). Brit. med. J., 1, 13. (1954b). Lancet, 2, 684 . 